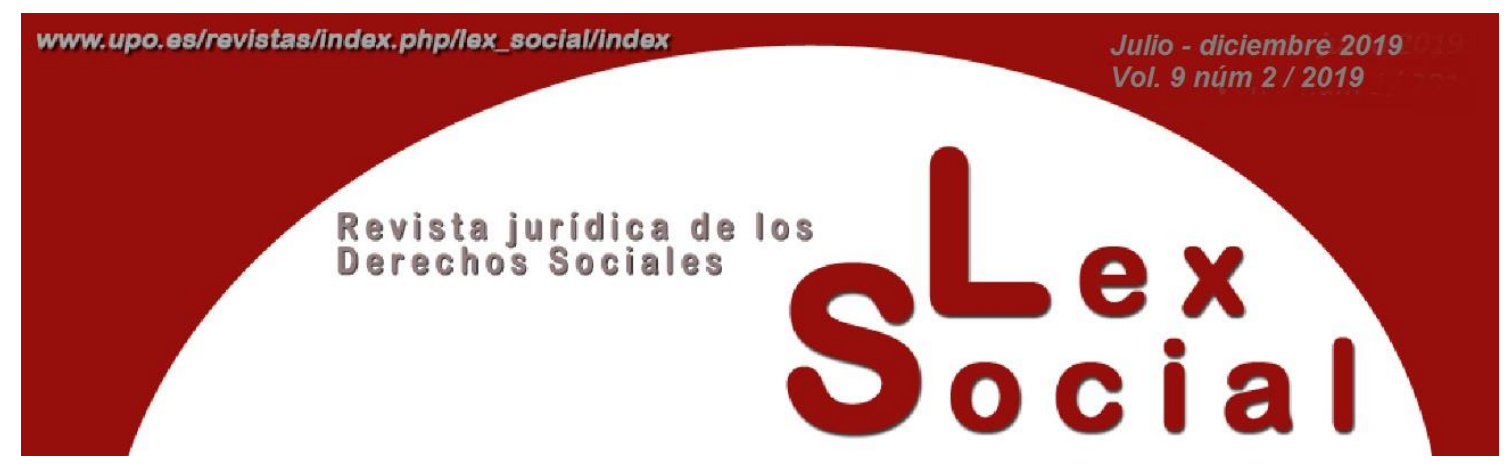

\title{
TRABAJO DOMÉSTICO Y DERECHOS COLECTIVOS. ALGUNAS REFLEXIONES AL HILO DEL RD 1620/2011 Y DEL CONVENIO 189 OIT
}

\author{
DOMESTIC WORK AND COLLECTIVE RIGHTS. SOME \\ REFLECTIONS TO THE THREAD OF RD 1620/2011 AND ILO \\ CONVENTION 189
}

\author{
PATRICIA NIETO ROJAS \\ Profesora Ayudante Doctor. \\ Universidad Carlos III de Madrid
}

Artículo recibido el 10 de mayo de 2019

Artículo aceptado el 27 de mayo de 2019

\section{RESUMEN}

La precariedad de muchas de las relaciones laborales que se amparan al servicio del hogar familiar dificulta la actividad sindical. partiendo de este hecho, en el presente artículo se valorarán la virtualidad de diferentes mecanismos para asegurar el ejercicio de derechos colectivos por las trabajadores domésticas, planteándose la idoneidad de atribuir esta representación ex lege a los sindicatos más representativos. en cierta medida, esta función ha sido asumida por ccoo y ugt en las diferentes mesas de diálogo social, exigiendo al gobierno la ratificación del convenio 189 oit. la ratificación de este convenio supondría un importante aliciente para una mejor protección de los derechos colectivos de las trabajadoras domésticas, pues no solo insta a los estados a adoptar medidas para asegurar que los trabajadores domésticos disfruten de la libertad sindical sino porque mandata a todos los estados a proteger el derecho de los trabajadores domésticos a constituir las organizaciones, federaciones y confederaciones que estimen convenientes. 
Palabras Clave: Trabajo doméstico; derechos colectivos; convenio 189 OIT, sindicatos; Grupo Turín.

\begin{abstract}
The precariousness of many of the labor relations that support the service of the family home hinders union activity. In this article, it is analyzed the role assumed by majority traditional trade union (CCOO and UGT) to asumme the employee representation in the social dialogue process. In the C189- Domestic Works Convention, the article 3 says that "Each Member shall, in relation to domestic workers, take the measures set out in this Convention to respect, promote and realize the fundamental principles and rights at work, namely: (a) freedom of association and the effective recognition of the right to collective bargaining and taking measures to ensure that domestic workers and employers of domestic workers enjoy freedom of association and the effective recognition of the right to collective bargaining, Members shall protect the right of domestic workers and employers of domestic workers to establish and, subject to the rules of the organization concerned, to join organizations, federations and confederations of their own choosing.
\end{abstract}

KEYWORDS: Domestic service, collective rights, social protection, Convention 189 ILO, trade unions; Turin group.

SUMARIO

1. A MODO DE INTRODUCCIÓN: LA PRECARIEDAD DEL TRABAJO DOMÉSTICO COMO PUNTO DE PARTIDA

2. EL EJERCICIO DE DERECHOS COLECTIVOS EN EL HOGAR FAMILIAR

2.1. LA ATRIBUCIÓN DE LA REPRESENTACIÓN A LOS SINDICATOS MÁS REPRESENTATIVOS

2.2. LA REPRESENTACIÓN DE LAS TRABAJADORAS DOMÉSTICAS POR SUJETOS NO RECONOCIDOS POR EL DERECHO SINDICAL

3. A MODO DE REFLEXIÓN FINAL

4. BIBLIOGRAFÍA CITADA 


\section{A MODO DE INTRODUCCIÓN: LA PRECARIEDAD DEL TRABAJO DOMÉSTICO COMO PUNTO DE PARTIDA}

El artículo 2.1 b) ET, considera, como bien es sabido, relación laboral de carácter especial la del servicio del hogar familiar, conteniéndose su regulación básica en el RD 1620/2011, de 14 de noviembre. Las condiciones particulares en que se realiza la actividad de las personas que trabajan en el servicio doméstico son, a juicio del referido $\mathrm{RD}$, las que justifican una regulación específica y diferenciada. De modo principal, el ámbito donde se presta la actividad, el hogar familiar, tan vinculado a la intimidad personal y familiar y por completo ajeno y extraño al común denominador de las relaciones laborales, que se desenvuelven en entornos de actividad productiva presididos por los principios de la economía de mercado; y, en segundo lugar y corolario de lo anterior, el vínculo personal basado en una especial relación de confianza que preside, desde su nacimiento, la relación laboral entre el titular del hogar familiar y los trabajadores del hogar, que no tiene que estar forzosamente presente en los restantes tipos de relaciones de trabajo. Interesándonos en este trabajo cuál es la regulación de los derechos colectivos, significadamente el derecho de sindicación, huelga y de representación legal para los trabajadores al servicio del hogar familiar ${ }^{1}$, conviene anticipar que el art. 7 RD 1620/2011 señala que los mismos tendrán "los derechos y deberes laborales establecidos en el presente RD y en los artículos 4 y 5 del Estatuto de los Trabajadores".

Partiendo de este reconocimiento, las trabajadoras domésticas serán titulares de los siguientes derechos básicos: b) Libre sindicación. c) Negociación colectiva. d) Adopción de medidas de conflicto colectivo. e) Huelga. f) Reunión. g) Información, consulta y participación en la empresa, con el contenido y alcance que para cada uno de los mismos disponga su específica normativa.

Ahora bien, sin perjuicio del reconocimiento normativo de estos derechos, nadie cuestiona que la precariedad laboral dificulta la actividad sindical. Basten, a este respecto, tres rasgos que caracterizan este tipo de ocupación:

En primer lugar, la parcialidad de muchas de estas contrataciones como evidencia el cuadro adjunto en el que, a partir de los datos proporcionados por el INE, se constata que el 55,15\% de las trabajadoras domésticas tienen empleos a tiempo parcial, siendo este porcentaje en cómputo general del 18,2\%.

\footnotetext{
${ }^{1}$ Deliberadamente se establecerá la expresión trabajadoras domésticas a la luz de la feminización del sector como se analiza detalladamente en otros apartados de este estudio.
} 


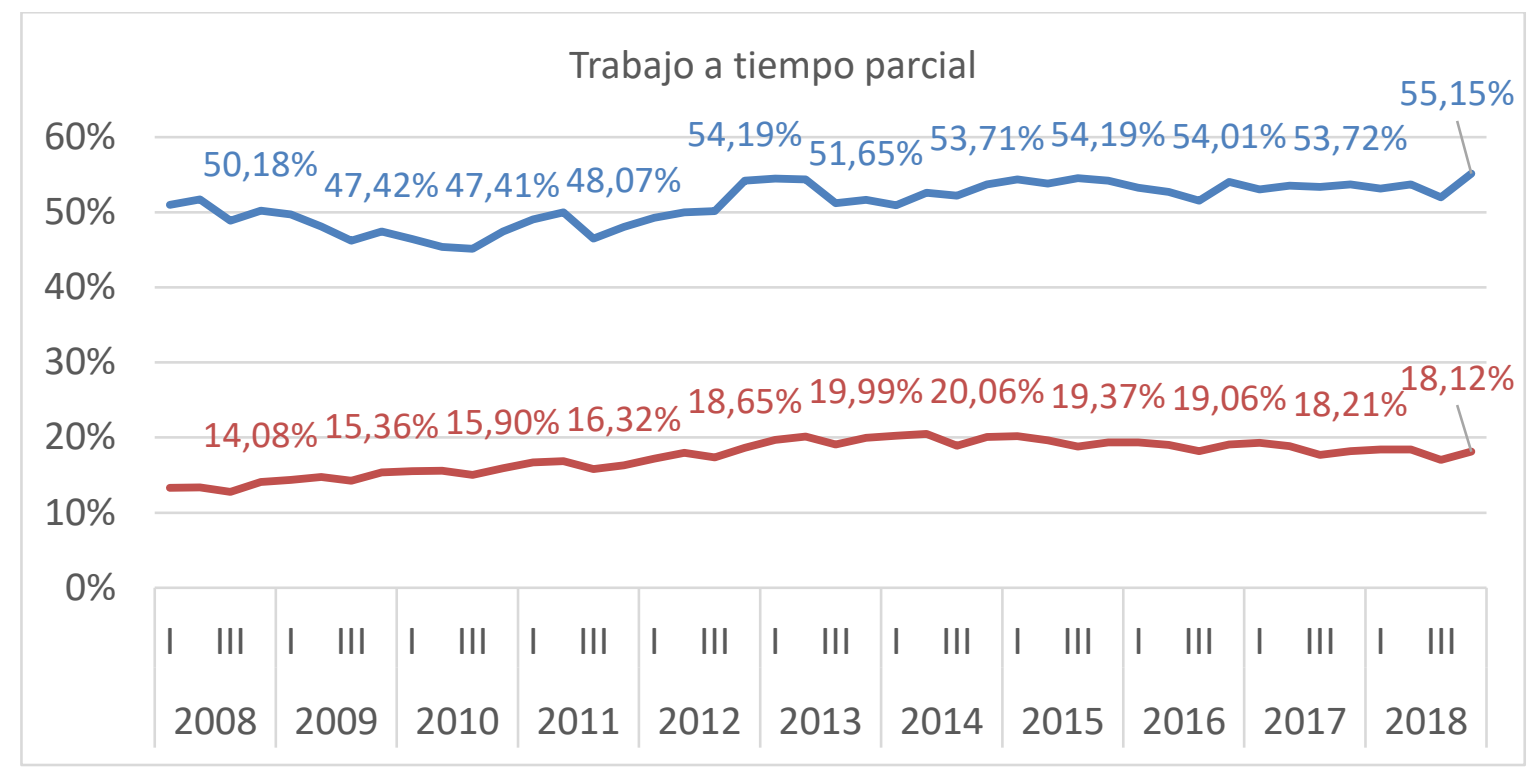

En segundo lugar, que el lugar de trabajo sea, al tiempo, el domicilio de las propias trabajadoras hace que estas vean mucho más debilitada, si cabe, su capacidad de acción colectiva.

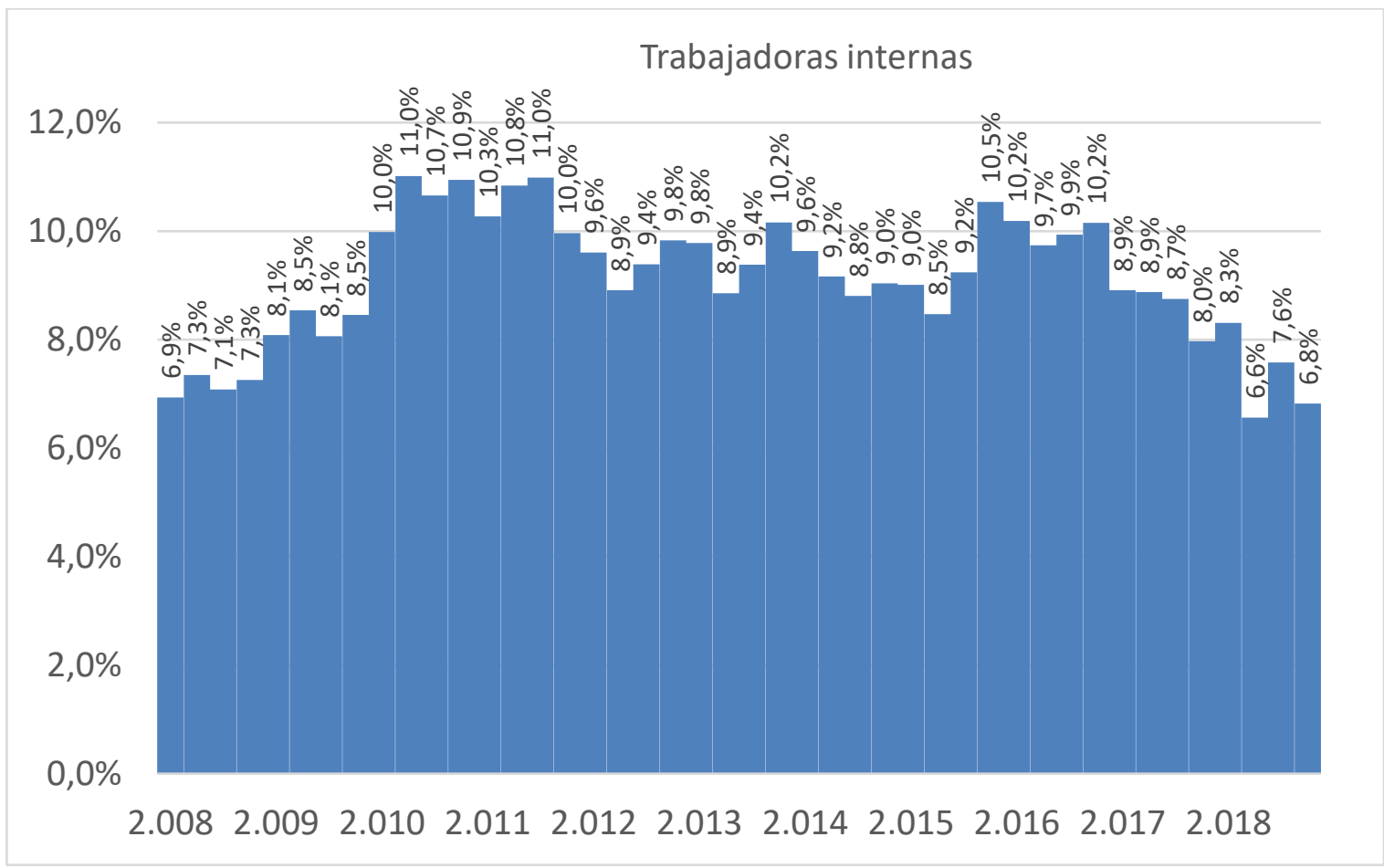

Y, en tercer lugar, este sector se caracteriza por un elevado porcentaje de trabajadoras inmigrantes (el mismo alcanza un $60 \%$ si atendemos a las trabajadoras domésticas que son extranjeras o que poseen doble nacionalidad) y bien es sabido que este factor también entraña una mayor dificultad para el ejercicio de derechos colectivos. 


\begin{tabular}{|c|c|c|c|c|c|}
\hline & \multicolumn{3}{|c|}{ Período de referencia } & \\
\hline & & \multicolumn{3}{|c|}{2018} & \\
\hline & & \multicolumn{3}{|c|}{ Actividad principal } & \\
\hline & & \multicolumn{3}{|c|}{$\begin{array}{l}\text { Actividades de los hogares como } \\
\text { empleadores de personal doméstico }\end{array}$} & \\
\hline & & \multicolumn{3}{|c|}{$\begin{array}{l}\text { Relación con la persona de } \\
\text { referencia }\end{array}$} & \\
\hline & & Externas & Internas & Total & \\
\hline \multirow[t]{4}{*}{ Nacionalidad } & Española & 268592 & 3894 & 272487 & $46 \%$ \\
\hline & $\begin{array}{l}\text { Española y } \\
\text { doble } \\
\text { nacionalidad }\end{array}$ & 67318 & 7358 & 74676 & $12 \%$ \\
\hline & Extranjera & 246890 & 34778 & 281669 & $42 \%$ \\
\hline & Total & 582801 & 46031 & 628832 & \\
\hline
\end{tabular}

La precariedad, irregularidad y desprotección social que caracteriza al empleo de este sector hace que el ejercicio de derechos colectivos por las trabajadoras domésticas sea escaso $^{2}$, cuando no existente, y esta anomia no deriva de la prohibición del ordenamiento jurídico nacional prohíba el ejercicio de derechos colectivos, que no lo hace. Es cierto que el RD 1620/2011 continúa, como la legislación anterior, sin hacer ninguna referencia a los derechos colectivos de estas trabajadoras, o, dicho de otro modo, ni los prohíbe, ni los reconoce ${ }^{3}$, ni los regula, lo que supone, en ciertos casos "un alto grado de dificultad que roza la imposibilidad de su ejercicio por las peculiaridades que se dan en esta relación especial" 4 .

Un reciente estudio de la OIT, denominado "Impacto de las reformas legislativas en el sector del empleo de hogar en España ${ }^{5 ",}$, recuerda que, a finales de 2016, había 630.100 personas ocupadas como personal doméstico -lo que representa el 3,4\% de las ocupadas- según la EPA del cuarto trimestre 2016, casi 9 de cada 10 personas trabajadoras en este sector son mujeres, en su mayoría migrantes. Además, la economía sumergida en este sector supera el 30\%, diez puntos porcentuales por encima de otros sectores. Respecto a las condiciones laborales, se estima que "el 57\% de las trabajadoras

\footnotetext{
${ }^{2}$ SANZ SÁEZ, C., Derechos colectivos y empleadas del hogar: reformas jurídicas inaplazables para un trabajo decente del trabajo doméstico en España, p. 5, fecha de consulta 6 mayo 2019, en https://www.ccoo.es/99d7ae8ee035fef57c16d423f2cb62eb000001.pdf.

3 RODRÍGUEZ SÁNCHEZ, R., La protección de los derechos colectivos en las relaciones laborales especiales, Rovira i Virgil, Tarrragona, 2004, p. 318.

${ }^{4}$ La evidente dificultad del ejercicio de derechos colectivos por las empleadas domésticas es unánimemente considerada por la doctrina. Véase, por todos, Cueva PuENTE, C., La relación laboral especial de los empleados de hogar, Lex Nova, Valladolid, 2005, p. 35.

${ }^{5}$ Impacto de las reformas legislativas en el sector del empleo del hogar en España / Oficina Internacional del Trabajo. - Ginebra: [OIT], 2016. (Conditions of work and employment series; No. 82, ISSN: 22268944; 2226-8952 (web pdf))
} 
no tienen marcado un límite sobre jornada laboral semanal, cerca del $45 \%$ tampoco tiene un día fijo o señalado para el descanso semanal, ni permisos por maternidad"6.

\section{EL EJERCICIO DE DERECHOS COLECTIVOS EN EL HOGAR FAMILIAR}

El Convenio de la OIT adoptado por la Conferencia el 16 de junio de 2011 (Convenio 189 y la Recomendación 201) es una medida de corte internacional destinada a mejorar las condiciones laborales de millones de trabajadores del sector, y aunque es evidente que la ratificación del convenio por el Estado español del convenio reforzaría la libertad sindical y el derecho a la negociación colectiva de estas trabajadoras, hay un buen número de factores extrajurídicos que dificultan el ejercicio de derechos colectivos.

No hay que olvidar que esta relación laboral se desarrolla en el domicilio de la parte empleadora, y este hecho determina "un aislamiento e invisibilidad de la parte empleada que dificulta enormemente la acción sindical, y si a ello le unimos una tradicional desatención de los sindicatos respecto a estas trabajadoras, podemos entender la ausencia total de negociación colectiva y que toda la regulación suele ser bastante dificultada. En este sentido, no podemos olvidar que existe un supuesto concreto en que el aislamiento e invisibilidad aumenta el riesgo de precariedad, que es este tipo de trabajo en régimen interno"7. De todo lo expuesto se puede inferir recordando que no es suficiente con considerar los derechos fundamentales como una pura referencia constitucional sino que "es necesario trasladar al plano normativo infraconstitucional la traducción de tales derechos fundamentales a los diferentes aspectos en él regulados"8 aunque cualquier solución que se proponga ha de partir de un dato: que el lugar de trabajo sea el hogar familiar no solo genera aislamiento para las trabajadoras domésticas con los retos que esta individualización supone para el hecho sindical, sino que, en muchas ocasiones, la actividad laboral se realiza sin compañía ni siquiera de quienes conviven en el hogar familiar.

La necesaria e imprescindible convivencia y cercanía entre trabajador y empleador, unidas a una dirección y control muy estrecho por parte de este último, que siempre ha de ser persona física, supone el mayor reto para el ejercicio de derechos colectivos, especialmente si tenemos en cuenta que, en este sector, no existe un colectivo laboral relativamente homogeneizado sino un conjunto fragmentado y desigual de trabajadoras. Entablar y desarrollar relaciones con una fuerza laboral dispersa puede ser un formidable obstáculo para la organización colectiva en la economía del trabajo doméstico. En demasiadas ocasiones, las empleadas domésticas, aisladas

\footnotetext{
${ }^{6}$ SANDE PÉREZ-BEDMAR, M., "La relación laboral especial de los empleados de hogar:aspectos laborales y de seguridad social”, Revista jurídica Universidad Autónoma de Madrid, vol. 23, 2011, p. 154.

${ }^{7}$ SÁNZ SÁEZ, C., Derechos colectivos y empleadas del hogar: reformas jurídicas inaplazables para un trabajo decente del trabajo doméstico en España, cit., p. 13.

8 RODRÍGUEZ SÁNCHEZ, R., La protección de los derechos colectivos en las relaciones laborales especiales, cit., p. 319. 
geográficamente, no tienen elementos de referencia que les sirvan para unirse con el fin de organizarse y actuar en defensa de sus intereses comunes, y es esta dispersión el mayor reto al que se enfrentan quiénes quieren asumir la representación legal de estas trabajadoras, sean estos sindicatos u otro tipo de plataformas.

Ante la evidente desprotección de este colectivo, cabría plantearse la idoneidad de atribuir esta representación ex lege a los sindicatos más representativos, tal y como ocurrió en relación a las empresas de trabajo temporal cuando se aprobó su normativa reguladora ${ }^{9}$. Esta representación ha sido parcialmente asumida por CCOO y UGT en las diferentes mesas de diálogo social, exigiendo al Gobierno la ratificación del convenio 189 OIT $^{10}$. Ahora bien, como seguidamente analizaremos, hay otros movimientos reivindicativos que también están pretendiendo capitalizar esta demanda (v. gr. Grupo Turín).

En la Unión Europea ha sido la EFFAT, Federación Europea de Sindicatos que agrupa a los Sectores de la Alimentación, la Agricultura y el Turismo, la que ha asumido esta función, comprometiéndose a apoyar a sus sindicatos afiliados y al conjunto del movimiento de los trabajadores domésticos en Europa, del modo siguiente:

- Abordando con la Unión Europea y los diferentes gobiernos estatales la necesidad de trasponer, cuanto antes, el contenido del Convenio de la OIT número 189 a las legislaciones nacionales ('ratificar'), modificando, en su caso, todas las directivas de la UE que entren en conflicto con el C-189 y garantizar que esa normativa se aplica de manera adecuada.

- Animando a los sindicatos afiliados a actuar activamente con sus propios gobiernos y llegar a un mayor número de trabajadores domésticos y sus propias organizaciones, allí donde las haya ${ }^{11}$.

\subsection{La atribución de la representación a los sindicatos más representativos}

Con buen criterio se ha cuestionado si las vías de acción del sindicato (al menos, en su configuración tradicional) pueden ser eficaces para la representación de las trabajadoras al servicio del hogar familiar, máxime cuando nos encontramos ante un trabajo

\footnotetext{
${ }^{9}$ En redacción dada al art. 13 Ley 14/1994, de 1 de junio, por la que se regulan las empresas de trabajo temporal (Artículo 13. Negociación colectiva. En ausencia de órganos de representación legal de los trabajadores, estarán legitimados para negociar los convenios colectivos que afecten a las empresas de trabajo temporal las Organizaciones sindicales más representativas, entendiéndose válidamente constituida la representación de los trabajadores en la Comisión negociadora cuando de ella formen parte tales Organizaciones).

${ }^{10}$ Sobre esta actividad puede consultarse las acciones impulsadas desde los dos sindicatos mayoritarias, accedido el día $7 / 5 / 2019$

http://www.ugt.es/convenio-189-oit (www.ccoo.es/bb66a7eb9c65bdcd7980bd4c5316c5a1000001.pdf
}

${ }^{11}$ MATHHER, C., Los trabajadores domésticos en Europa se organizan, EFFAT, Bruselas, 2015, p. 5. ISSN: $2174-6419$

Lex Social, vol. 9, núm. 2 (2019) 
"fragilizado" 12 en derechos. Ante la dificultad que plantea la asunción de esta representación, los sindicatos tradicionales (significadamente CCOO, UGT y USO) han impulsado una importante amalgama de actividades en pos de la protección de este colectivo y para ello han impulsado no solo funciones de representación sino también las que corresponden al sindicato como agente institucional del mercado de trabajo ${ }^{13}$, tanto a través de acciones de capacitación profesional como, en su caso, asumiendo funciones de intermediación laboral. Lamentablemente los sindicatos no han podido avanzar en la necesaria regulación sectorial de las condiciones de trabajo de este colectivo, aunque es evidente que negociación colectiva cumplirá un importante papel corrector y nivelador de la situación de desequilibrio en que se encuentran las partes de la relación, modificando el esquema esencialmente contractualista de la misma por un planteamiento colectivo de sus intereses.

Entre las razones que pueden explicar la inexistencia de esta negociación sectorial no solo derivan de la escasa movilización colectiva de estas trabajadoras sino que se justifican por la ausencia de contraparte empresarial con la que negociar, dada la inexistencia de asociaciones de titulares del hogar familiar, siendo además poco probable que la situación cambie porque el empleador, siempre persona física, no necesita de la asociación ni de la negociación ${ }^{14}$ aunque, en un plano teórico, sí que existirían fórmulas para solventar esta disfunción:

En primer lugar, cabría la fijación de condiciones laborales para el conjunto de los trabajadores del hogar familiar en un ámbito territorial determinado a través del acto de extensión de un convenio colectivo, con características económico-laborales equiparables, en atención a la previsión contenida en el art. 92.2 ET, aunque convendremos en lo poco probable que esta opción se materialice en la práctica.

En segundo lugar, que el propio art. 3 c) RD 1620/2011 haga una referencia expresa a los convenios colectivos como fuente de regulación laboral podría servir para defender la posible aplicación de algún convenio a las trabajadoras domésticas, aunque lo cierto es que solo se ha localizado un convenio colectivo ${ }^{15}$ que incluye en el ámbito de aplicación "los domicilios particulares". En él se establece que si la titularidad del hogar familiar "contrate cualquiera de las actividades descritas con empresas del sector, con la excepción de aquellos artículos que puedan afectar a la inviolabilidad del domicilio, a la intimidad familiar y personal o a la responsabilidad establecidas para los titulares del hogar familiar, recogidas como relaciones laborales de carácter especial en el artículo 2 del Estatuto de los trabajadores, y las exclusiones igualmente contempladas en el artículo 1.3 del mismo cuerpo legal".

\footnotetext{
${ }^{12}$ Expresión utilizada por BAYLOS GRAU, A., "La acción sindical ante el fenómeno de la economía sumergida", en Monereo Pérez, J. L., Perán Quesada, S. (eds.) Derecho social y trabajo informal: implicaciones laborales, económicas y de Seguridad Social del fenómeno del trabajo informal y de la economía sumergida en España y Latinoamérica, Comares, Granada, 2016, p. 599.

${ }^{13}$ Véanse las diferentes acciones impulsadas por USO (https://www.uso.es/tag/empleo-domestico/)

${ }^{14}$ RodríGUeZ SÁNCHEZ, R., La protección de los derechos colectivos en las relaciones laborales especiales, cit., p. 327.

${ }^{15}$ CC Limpieza Edificios y locales Cataluña, DOGC 3.9.2018.
} 
Dos son las razones que, a mi juicio, explican la ausencia de negociación colectiva en este sector:

En primer lugar, el escaso poder negociador de la parte social, habida cuenta que la afiliación sindical es muy escasa, por no decir nula, y, en segundo lugar, la inexistencia de asociaciones empresariales específicas. La singularidad del titular del hogar familiar (que, en ningún caso, está asociado a asociaciones empresariales que pudieran representarlos), y del propio trabajador (que normalmente es uno solo por cada hogar familiar $y$, en consecuencia, excluido de toda forma de representación electiva o unitaria, que es la base de la legitimación para negociar convenios en los términos del Título II del ET) han impedido la suscripción de normas colectivas sectoriales de aplicación general.

Ahora bien, conocida la ausencia de regulación sectorial, la DA $7^{\text {a }}$ ET contemplaba, a este respecto, una solución de "emergencia", concretamente preveía "la regulación de condiciones de trabajo por rama de actividad para los sectores económicos de la producción y demarcaciones territoriales en que no exista convenio colectivo podrá realizarse por el Gobierno, a propuesta del Ministerio de Empleo y Seguridad Social, previas las consultas que considere oportunas a las asociaciones empresariales y organizaciones sindicales, sin perjuicio de lo dispuesto en el artículo 92, que será siempre procedimiento prioritario" aunque, como bien es sabido, nunca se ha explorado la virtualidad de esta fórmula.

Más recientemente, el RD Ley 7/2011, de 10 de junio, de medidas urgentes para la reforma de negociación colectiva ${ }^{16}$, trató de establecer una solución para "aquellos sectores en los que no existan órganos de representación de los trabajadores". Para ello, se dio una nueva redacción al art. 88.2 ET, de modo que "se entenderá válidamente constituida la comisión negociadora cuando la misma esté integrada por las organizaciones sindicales que ostenten la condición de más representativas en el ámbito estatal o de comunidad autónoma".

En el sector que ahora ocupa, serían los sindicatos más representativos de ámbito estatal o de comunidad autónoma, según los casos, los que ostentarían la legitimación plena para suscribir los pertinentes convenios colectivos de sector.

En relación a la representación empresarial, los problemas de legitimación se tratan de solventar con la nueva redacción dada al art. 87 ET, apartado 3 c), último párrafo, al entender que «en aquellos sectores en los que no existan asociaciones empresariales que cuenten con la suficiente representatividad (...) estarán legitimadas para negociar los correspondientes convenios colectivos de sector las asociaciones empresariales de ámbito estatal que cuenten con un 10 por ciento o más de las empresas o trabajadores en 
el ámbito estatal, así como las asociaciones empresariales de comunidad autónoma que cuenten en ésta con un mínimo del 15 por ciento de las empresas o trabajadores». De manera semejante a lo que sucede respecto de la representación sindical, la imposibilidad o ausencia de legitimación sectorial concreta queda suplantada por la mayor representatividad a nivel estatal o autonómico. Por último, y en lo que se refiere a la legitimación plena para constituir válidamente la comisión negociadora, la nueva redacción del art. 88.2 ET establece que: «en aquellos sectores en los que no existan asociaciones empresariales que cuenten con la suficiente representatividad, se entenderá válidamente constituida la comisión negociadora cuando la misma esté integrada por las organizaciones empresariales estatales o autonómicas referidas en el párrafo segundo del artículo 87.3, c)».

La reforma de la negociación colectiva introducida por el RDL 7/2011 permitiría la negociación de convenios sectoriales (que serían los únicos realmente posibles para los trabajadores al servicio del hogar familiar) por parte de sindicatos y organizaciones empresariales más representativos, sean estatales o de comunidad autónoma; incluso cuando en el sector en cuestión no existan sujetos que reúnan los requisitos generales de legitimación exigidos con carácter general en los arts. 87 y 88 ET (que es lo que sucede en el caso de la relación laboral del servicio del hogar familiar $)^{16}$.

Casi de manera profética se advertía de que la posibilidad de negociar "un convenio colectivo, estatal o autonómico, referido a las trabajadoras domésticos no significa que éste vaya a existir necesariamente ya que se requiere que tanto los sindicatos más representativos como las asociaciones empresariales más representativas también sean proclives a su existencia; todo ello sin olvidar el escaso margen que al respecto ha dejado el propio RD 1620/2011 ${ }^{17}$,.

Contrasta la situación española con la experiencia existente en otros estados europeos donde la negociación colectiva sectorial ${ }^{18}$ cuenta con una dilatada trayectoria. A este respecto, en Italia, fueron cuatro sindicatos FILCAMS, FISASCAT, UILTuCS y FEDERCOLF) y dos asociaciones patronales (FIDALDO y DOMINA) los que suscribieron un convenio sectorial ${ }^{19}$. También en Alemania existe "una larga tradición de negociación colectiva entre el sindicato NGG y la organización de empleadores DHB NetzwerkHaushalt (Red de hogares)", que data de los pasados años 50 y aunque "estos convenios solo se aplican a los afiliados de DHB y NGG, lo que supone un número relativamente reducido de trabajadores domésticos", se ha defendido que "son un modelo a seguir" 20 . Finalmente, en Francia, la FEPEM (Fédération des Particuliers

\footnotetext{
17 BARCELón COBEDO, S., "La relación laboral del servicio del hogar familiar tras el RD 1620/2011, de 14 de noviembre.", Aranzadi Social, vol. 1/2012, BIB 2012 1512, p. 6.

${ }^{18}$ Sobre las diferentes experiencias convencionales para regular colectivamente el trabajo doméstico en Europa puede verse MATHHER, C., Los trabajadores domésticos en Europa se organizan, cit., pp. 27-29.

19 El texto del convenio puede consultarse en (http://www.filcams.cgil.it/lavoro-domestico-ccnl-1-072013-31-12-2016-testo-ufficiale/) Fecha de acceso: 8/5/2019

${ }^{20}$ MATHHER, C., Los trabajadores domésticos en Europa se organizan, cit., p. 30. ISSN: 2174-6419

Lex Social, vol. 9, núm. 2 (2019)
} 
Employeurs de France), organización patronal que representa a los propietarios de las casas particulares que contratan directamente a un trabajador doméstico, suscribió un convenio colectivo con las federaciones de sindicatos CFDT, CGT, CFTC y FO que data del año 1999 y que fue pieza instrumental en la creación del programa de vales CESU.

\subsection{La representación de las trabajadoras domésticas por sujetos no reconocidos por el Derecho Sindical}

$\mathrm{Al}$ igual que ocurre en el sector de las plataformas digitales, donde en todos los países ${ }^{21}$ están apareciendo movimientos colectivos, muchos de corte asambleario ${ }^{22}$, que pretenden dar una respuesta articulada a las reivindicaciones laborales de los prestadores de servicios en plataformas, también en el sector del trabajo doméstico emergen nuevos actores que presenten capitalizar esta representación de intereses colectivos. Antes de proceder a sistematizar este tipo de fórmulas, conviene precisar que en el presente trabajo asumimos la noción de movilización colectiva defendida en el informe de la OIT, "Representación, voz y negociación colectiva: la sindicalización en la economía del trabajo esporádico y por encargo", entendiendo como tal la decisión de "actual conjuntamente para maximizar su capacidad de ejercer influencia y provocar un cambio" 23 .

El movimiento más activo dentro de este sector lo representa el "Grupo Turín" 24 que es una plataforma creada en el año 2012, compuesta por entidades y personas que, desde diferentes ámbitos y perspectivas, y que trabaja por la dignificación del sector denominado empleo del hogar y los cuidados. Su principal actuación en la actualidad es conseguir que el Estado Español ratifique el convenio 189 de la OIT (y recomendación 201).

También ha asumido esta función de representación de intereses colectivos la asociación SEDOAC "Servicio doméstico activo", que se define a sí misma como una "asociación de mujeres de diferentes nacionalidades que cansadas de pertenecer a un sector invisible, vulnerable y precario decidimos unir nuestras fuerzas y luchar por la igualdad plena de los derechos: laborales, políticos, sociales y civiles de todas las trabajadoras de hogar en España así como la reivindicación de la igualdad plena de derechos y de sus condiciones justas y dignas; con ámbito nacional e internacional" 25 .

Aunque es evidente que estos movimientos asumen una función parasindical, los mismos tienen, desde la perspectiva jurídico-formal, un alcance muy limitado, al menos, en el ordenamiento jurídico español, pues estas asociaciones no tienen competencias y

${ }^{21}$ Trillo PÁRragA, F., "Economía digitalizada y relaciones de trabajo", Revista de Derecho Social, vol. 76, 2016, p. 80. Sobre las diferentes iniciativas impulsadas: http://faircrowd.work/unions-forcrowdworkers/

22 Una completísima síntesis de este tipo de fórmulas en JHONSTON, H.; LAND-KAZLAUSKAS, C., Representación, voz y negociación colectiva: la sindicalización en la economía del trabajo esporádico y por encargo, ILO, Ginebra, 2018, pp. 12-15.

${ }^{23}$ Véase nota 1 Ibid., p. 1.

${ }^{24} \mathrm{http}$ ://congresoempleodehogarycuidados.es/grupo-turin-quienes-somos/

${ }^{25} \mathrm{http}$ ://serviciodomesticoactivo.blogspot.com/p/quienes-somos.html ISSN: $2174-6419$

Lex Social, vol. 9, núm. 2 (2019) 
funciones legalmente atribuidas lo que impide su consideración como sujeto representativo propio al menos, en la lógica, del modelo sindical español. Evidentemente, la legitimidad de su actuación se puede justificar en base a la previa elección de sus compañeros aunque la eficacia de su actuación se circunscribe a la acreditación de una representación privada, pues no existe en nuestro ordenamiento ninguna norma legal que conceda a estos representantes asamblearios un poder para pactar acuerdos con la especial eficacia que el Estatuto de los Trabajadores otorga a los pactos alcanzados por representaciones legales ${ }^{26}$. O, dicho de otro modo, nuestra legislación laboral, en ningún caso, posibilita la figura del representante asambleario y es dudoso que esta figura pueda ejercer funciones de representación vinculantes para el conjunto de los trabajadores, fuera de los límites generales del Derecho Privado.

\section{A MODO DE REFLEXIÓN FINAL}

La mejor forma de conseguir la dignificación de las condiciones laborales de las trabajadoras exige de una actuación coordinada que actúe en plano simultáneos:

En primer lugar, es necesaria una actuación pública que ratifique, por fin, el convenio 189 OIT. Sin embargo, como recientemente ha dicho la profesora Grau Pineda ${ }^{27}$ "da la sensación de tratarse de un convenio que ha caído en el olvido y que, salvo la insistencia recurrente del todavía incipiente movimiento asociativo, ha sido imbuida por una desidia que imposibilita la necesaria acción política. Que España podría haber ratificado el Convenio 189 desde el día siguiente a su aprobación y haber llevado a cabo las modificaciones/adaptaciones normativas precisas sin solución de continuidad, nadie lo pone en duda. Pero no se ha optado por esa posibilidad y, en estos momentos de impasse, conviene insistir en que la ratificación del Convenio 189, además de ser una cuestión de justicia social, es importante también en términos económicos y de seguridad social".

En segundo lugar, es necesario que trabajadoras domésticas se organicen colectivamente y reciban el apoyo del movimiento sindical para hacerlo. Sin embargo, son muchos los retos a los que esta acción colectiva se enfrenta no solo porque existen distintos tipos de trabajadores domésticos (a tiempo completo o parcial, trabajo para un único o varios hogares, contratados por hogares particulares o por empresas, de origen inmigrante o nacional, etc.) sino porque muchas de estas trabajadoras se encuentran aisladas, lejos de sus seres queridos, sus comunidades y otros trabajadores como ello.

A menudo, estas trabajadoras tienen miedo de contactar con los sindicatos por temor a perder sus trabajos. Por ello, y con el objetivo último de mejorar su situación, se ha entendido que el primer paso es generar materiales informativos para que tomen

\footnotetext{
${ }^{26}$ LIMÓN LUQUe, M. A., Derecho de reunión y relaciones laborales: el derecho de asamblea en la empresa, CES, Madrid, 1996, pp. 129, 348.

27 https://www.laprovincia.es/sociedad/2019/05/05/sirvientas-trabajadoras/1171822.html Accedido el 9/5/2019.
} 
conciencia de la situación (significadamente mediante páginas web, vídeos de YouTube, o páginas de Facebook que puedan compartir entre ellos) aunque el contacto personal también es sumamente importante, sobre todo para estos trabajadores aislados. Eso significa acudir a los sitios donde los trabajadores se reúnen, y para llegar a aquéllos que apenas, incluso nunca, asisten a reuniones.

Los sindicatos que, durante mucho tiempo, se han centrado en una organización colectiva en el lugar de trabajo, descubren que tienen que "regresar a sus orígenes", saliendo a la comunidad y trabajando de modo interpersonal ${ }^{28}$.

\section{BIBLIOGRAFÍA CITADA}

BARCELÓN COBEDO, S., "La relación laboral del servicio del hogar familiar tras el RD 1620/2011, de 14 de noviembre.”, Aranzadi Social, vol. 1/2012, n. ${ }^{\circ}$ BIB $2012 \backslash 512$.

BAYLOS GRAU, A., "La acción sindical ante el fenómeno de la economía sumergida", en Monereo Pérez, J. L., Perán Quesada, S. (eds.) Derecho social y trabajo informal: implicaciones laborales, económicas y de Seguridad Social del fenómeno del trabajo informal y de la economía sumergida en España y Latinoamérica, Comares, Granada, 2016.

CUEVA PUENTE, C., La relación laboral especial de los empleados de hogar, Lex Nova, Valladolid, 2005.

JHONSTON, H.; LAND-KAZLAUSKAS, C., Representación, voz y negociación colectiva: la sindicalización en la economía del trabajo esporádico y por encargo, ILO, Ginebra, 2018.

LIMÓN LUQUE, M. A., Derecho de reunión y relaciones laborales: el derecho de asamblea en la empresa, CES, Madrid, 1996.

MATHHER, C., Los trabajadores domésticos en Europa se organizan, EFFAT, Bruselas, 2015.

RODRÍGUEZ SÁNCHEZ, R., La protección de los derechos colectivos en las relaciones laborales especiales, Rovira i Virgil, Tarrragona, 2004.

SANDE PÉREZ-BEDMAR, M., "La relación laboral especial de los empleados de hogar:aspectos laborales y de seguridad social", Revista jurídica Universidad Autónoma de Madrid, vol. 23, 2011.

${ }^{28}$ MATHHER, C., Los trabajadores domésticos en Europa se organizan, cit., p. 8. 
SÁNZ SÁEZ, C., Derechos colectivos y empleadas del hogar: reformas jurídicas inaplazables para un trabajo decente del trabajo doméstico en España, fecha de $\begin{array}{lccc}\text { consulta } & 6 & \text { mayo } & 2019 \text {, } \\ \text { https://www.ccoo.es/99d7ae8ee035fef57c16d423f2cb62eb000001.pdf. }\end{array}$

TRILLO PÁRRAGA, F., "Economía digitalizada y relaciones de trabajo", Revista de Derecho Social, vol. 76, 2016. 